\title{
The global dissemination of hospital clones of Enterococcus faecium
}

\author{
Sebastiaan J. van Hal ${ }^{1,2^{*}}$ D, Rob J. L. Willems ${ }^{3}$, Theodore Gouliouris ${ }^{4}$, Susan A. Ballard ${ }^{5}$, Teresa M. Coque ${ }^{6,7}$, \\ Anette M. Hammerum ${ }^{8}$, Kristin Hegstad ${ }^{9,10}$, Hendrik T. Westh ${ }^{11}$, Benjamin P. Howden ${ }^{5}$, Surbhi Malhotra-Kumar ${ }^{12}$, \\ Guido Werner ${ }^{13}$, Katsunori Yanagihara ${ }^{14}$, Ashlee M. Earl ${ }^{15}$, Katherine E. Raven ${ }^{16}$, Jukka Corander ${ }^{17,18}$, \\ Rory Bowden ${ }^{19,20,21}$ and Enterococcal Group
}

\begin{abstract}
Background: The hospital-adapted A1 group of Enterococcus faecium remains an organism of significant concern in the context of drug-resistant hospital-associated infections. How this pathogen evolves and disseminates remains poorly understood.

Methods: A large, globally representative collection of short-read genomic data from the hospital-associated A1 group of Enterococcus faecium was assembled $(n=973)$. We analysed, using a novel analysis approach, global diversity in terms of both the dynamics of the accessory genome and homologous recombination among conserved genes.
\end{abstract}

Results: Two main modes of genomic evolution continue to shape E. faecium: the acquisition and loss of genes, including antimicrobial resistance genes, through mobile genetic elements including plasmids, and homologous recombination of the core genome. These events lead to new clones emerging at the local level, followed by the erosion of signals of clonality through recombination, and in some identifiable cases producing new clonal clusters. These patterns lead to new, emerging lineages which are able to spread globally over relatively short timeframes.

Conclusions: The ability of A1 E. faecium to continually present new combinations of genes for potential selection suggests that controlling this pathogen will remain challenging but establishing a framework for understanding genomic evolution is likely to aid in tracking the threats posed by newly emerging lineages.

\section{Background}

Enterococcus faecium, a commensal of the gastrointestinal tract, is a common cause of serious hospitalassociated infections [1]. Therapy is complicated by resistance to multiple antibiotics, including vancomycin; as a consequence, the World Health Organization includes vancomycin-resistant $E$. faecium (VRE) on its list of priority multidrug-resistant pathogens. E. faecium tends to persist in the hospital environment, leading to outbreaks

\footnotetext{
* Correspondence: Sebastiaan.vanhal@health.nsw.gov.au

'Department of Infectious Disesase and Microbiology, Royal Prince Alfred Hospital, Sydney, NSW, Australia

2University of Sydney, Sydney, NSW, Australia

Full list of author information is available at the end of the article
}

without clear transmission chains and the dissemination of antimicrobial resistance from a variety of sources [2], making the control of hospital E. faecium challenging.

Vancomycin resistance occurs in $30-50 \%$ of isolates in some countries and is considered the greatest threat to successful treatment. Vancomycin resistance is almost always linked to the presence of the $v a n A$ and/or $v a n B$ gene cluster, whose relative frequencies vary in space and time [3, 4]. Optimal therapy for VRE infections remains uncertain; daptomycin and linezolid are the most commonly utilised last-line antibiotics. Besides plasmidmediated linezolid resistance leading to sporadic outbreaks, resistance to last-line antibiotics remains

(c) The Author(s). 2021 Open Access This article is licensed under a Creative Commons Attribution 4.0 International License, which permits use, sharing, adaptation, distribution and reproduction in any medium or format, as long as you give appropriate credit to the original author(s) and the source, provide a link to the Creative Commons licence, and indicate if changes were made. The images or other third party material in this article are included in the article's Creative Commons licence, unless indicated otherwise in a credit line to the material. If material is not included in the article's Creative Commons licence and your intended use is not permitted by statutory regulation or exceeds the permitted use, you will need to obtain permission directly from the copyright holder. To view a copy of this licence, visit http://creativecommons.org/licenses/by/4.0/. The Creative Commons Public Domain Dedication waiver (http://creativecommons.org/publicdomain/zero/1.0/) applies to the data made available in this article, unless otherwise stated in a credit line to the data. 
uncommon [5]. Mutations in any of three genes (liaF, liaS, liaR) linked to cell wall stress responses, or in genes for cardiolipin synthase ( $c l s)$ or glycerophosphoryl diester phosphodiesterase $(g d p D)$, may confer daptomycin resistance, while mutations in 23S rRNA, the Cfr rRNA methyltransferase gene or optrA may lead to linezolid resistance $[6,7]$. It remains unclear whether these resistance mutations arise preferentially on particular gene sequences or are associated with specific circulating clones.

Isolates of E. faecium can be placed in two genomically distinguishable groups, also referred to in the literature as "lineages" or "clades": a hospital-associated lineage (clade A) and a community-associated lineage (clade B) [8]. Clade A is further split into clade A1, represented by human clinical isolates, and several non-A1 sub-clades [9] that, together with clade B isolates, are rarely found in hospitalised patients [10]. Previous genomic studies of A1 E. faecium have revealed substantial levels of genome plasticity and evidence of clonal outbreaks in individual hospitals [2]. At the scale of national or regional surveillance, patterns of A1 evolution become more complex; for example, new clone(s) have been reported that have outcompeted and ultimately replaced existing clones (e.g. vanA ST1421 in Australia or vanB ST192 in Germany) [3, 4]. How local institutional and regional factors, including infection control and antimicrobial stewardship, shape the global population structure of hospital E. faecium, and specifically clade A1, remains uncertain.

Standard genomic analyses of bacterial evolution employ phylogenetic modelling of mutations occurring in the core genome, identified either by mapping to a reference or identifying SNPs in core genes in de novo assemblies. Both approaches disregard much of the information that impacts inferences of genomic relationships. In addition, when substantial ongoing recombination is the main driver of genomic diversity, phylogenetic methods fail to accurately capture coancestry relationships, i.e., they fail to link genomes that share the most recent ancestors for the largest proportion of their genome (and may instead group together genomes which have independently acquired a similar recombinant sequence) $[11,12]$. Accordingly, we adopted a combined analysis approach to examine the population structure and evolutionary dynamics of this important hospital pathogen by using similarity in gene content and proximity in co-ancestry to recover genomic relationships. Our approach attempts to maximise the utilisation of genomic information for a large, globally representative collection of A1 E. faecium isolate sequences. It defines genomic relationships based on the presence or absence of genes within the pan-genome, with subsequent fine-tuning based on admixture patterns of core-genome SNPs. These data have allowed us to investigate the size and structure of the Group A1 pan-genome, the population structure that has formed during its worldwide dissemination, and signatures of repeated acquisition of antimicrobial resistance, providing a new description of the mechanisms involved in the ongoing evolution of a globally significant pathogen.

\section{Methods}

\section{Defining A1 group isolates}

Short-read sequence data from a total of 1100 E. faecium isolates were contributed by study investigators. Of these, 321 were newly sequenced and uploaded to NCBI under project number PRJNA63689 [13], with the remaining sequences $(n=774)$ downloaded from NCBI. Five isolates failed quality checks (using fastQC v0.11.9) after adapter trimming using trimmomatic v0.38 [14] and were excluded. To enable previous grouping, the dataset was supplemented with 52 [clade A1 $(n=14)$, clade A2 $(n=28)$ and clade B $(n=10)]$ additional sequences from Lebreton et al. [8]. Reads were mapped to Aus0004 (a closed annotated Australian genome, GenBank: CP003351) using bwa [15]. Single nucleotide polymorphisms (SNPs) were identified in each isolate using FreeBayes [16] with alleles filtered for read depth $(>20)$, and mapping quality, requiring $90 \%$ of reads to support a variant allele call. The final SNP matrix included only variant sites present in $>75 \%$ of isolates. Using hierBAPS [17] with 2 levels of hierarchy and maxK of 20, isolates were assigned to A1 $(n=997), \mathrm{A} 2(n=109)$ and B $(n=$ 41) groups [8]. Isolate details and associated metadata can be found in Additional file 1: Table S1.

\section{Pan-genome analysis and clustering of A1 hospital- associated isolates}

Non-enterococcal reads, based on taxonomic k-mer matches using kraken2 [18], were discarded to prevent subsequent spurious gene calls as a result of contamination. Sequences were assembled using SPAdes v3.13.1 [19] under the "careful" option, with assemblies failing quality metrics excluded $(n=24)$. Contigs $<2000 \mathrm{bp}$ were removed and discarded from individual assemblies, which were annotated using prokka v1.13 [20] prior to pan-genome discovery using panaroo v1.1.2 [21] under the "sensitive" mode. This setting is optimised to find all genes present in all isolates with homologous genes classified when gene sequences differ by $>5 \%$ between isolates. The identified panaroo core-genome was larger than previous estimates and therefore the analysis was duplicated using Roary [22].

The panaroo output, a gene presence-absence matrix, was used to cluster isolates after initial dimension reduction using Barnes-Hut t-Distributed Stochastic Neighbour Embedding in $\mathrm{R}$ with the Rtsne v0.15 package 
https://github.com/jkrijthe/Rtsne [23]. The optimal number of clusters was evaluated with aid of the fviz nbclust function within the R factoextra v1.0.6 package https://github.com/kassambara/factoextra [24].

Subsequently, cluster membership was refined using the ChromoPainter tool embedded in FineSTRUCTURE v2.1.3 [25] with a starting mean recombination rate of $2.3 \times 10^{-7}$. This method models each genome as a succession of haplotype fragments from other genomes in the collection and assigns the closest matching sequence as the donor haplotype with switches between donors occurring at ancestral recombination breakpoints. All these genome sections can then be ascribed or "painted" according to their inferred origins among groups of related donor sequences. Isolates with similar admixture patterns across the majority of their genome, representing a continuous line of ancestry, signify specific lineages. These lineages coalesce to form clusters with isolates expected to share the greatest proportion (always $>35 \%$ ) of their co-ancestry with another isolate assigned to the same cluster. Conversely, highly admixed isolates, as a result of numerous recombination events, could not be assigned to any pan-genome assigned cluster and were excluded from further analysis. For admixed regions, the likely "donor" isolate (and by inference the country of origin) can be found by looking for the isolate that contains the most similar sequence in the alternative clusters.

Final refined clustering of A1 isolates can be found in Additional file 1: Table S1.

\section{Phylogenetic and spatial analysis}

Maximum clade credibility trees were constructed on cluster-specific SNP matrices, excluding isolates with unknown collection dates, following masking of identified recombination sites using ClonalFrameML [26]. For each relevant cluster, several population models (constant, exponential and skyline) in combination with various clock models (strict, relaxed exponential and random) were run for $3 \times 10^{8}$ iterations using BEAST v2.6.1 [27]. Resulting trees were pruned, and a maximum clade credibility tree was obtained from the optimally performing model.

A spatial analysis was performed for Cluster 2, with inclusion of a geographical trait based on latitude and longitude co-ordinates and phylogenetic nodes assigned to a geographical location based on ancestral state reconstruction. Transmission links for isolates and nodes were inferred using genomic, spatial and temporal variables and visualised using SpreaD3 [28] under a discrete trait model. All isolates and nodes are represented by a network of connecting lines depicting the likely "origin" and "destination". Lines are coloured according to "destination" location.
MLST, resistome, virulome and plasmidome analysis

Multi-locus sequence typing was performed using the de novo assemblies. Isolate resistomes were predicted using AMRfinder specifying E. faecium [29]. Mutation-based resistance for daptomycin was predicted by annotation of variants against the Aus0004 genome. A medianjoining network of the liaFSR genes was constructed using NETWORK v10.0 [30]. 23S rRNA-based linezolid resistance was predicted as previously described [31]. The virulome was predicted using blastn against the downloaded virulence factor database [32]. To determine genes likely associated with plasmids, we implemented mlplasmids [33] using de novo assemblies with a posterior probability cut-off of 0.7 .

\section{Statistical analysis}

Statistical analyses for associations were performed using the appropriate two-sided statistical test following consideration of the data distribution. A $p$ value $<0.05$ was considered significant, with all calculations performed using the R stats package $\mathrm{v}$ 3.4.4 [34].

\section{Results \\ Defining group A1 isolates in a global collection of hospital E. faecium}

Groups that had previously published E. faecium genome data were invited to contribute. Participants were requested to provide short-read sequence data representing randomly selected isolates, spread across time, from collections of vancomycin-susceptible and vancomycin-resistant $E$. faecium originating from hospitalised patients in any country, ensuring that isolates from the same hospital were not enriched for known outbreaks, based on local epidemiological data.

Sequence variation identified through mapping reads to a reference genome was used to cluster isolates with hierBAPS [17], recovering the previously published A1 $(n=997), \mathrm{A} 2(n=109)$ and B $(n=41)$ groups. Of the A1 isolates, 24 subsequently failed de novo assembly quality filters and were excluded, leaving 973 A1 isolates originating from 31 countries, spanning a 30-year period (1986-2016) for analysis in this study.

\section{Heterogeneity among group A1 hospital E. faecium isolates}

To study the relationships among E. faecium A1 isolates, a pan-genome analysis based on de novo-assembled genomes, investigating large-scale variation comprising the presence and absence of genes and distinct forms of genes, was undertaken using Panaroo [21]. The 973 A1 isolates were separated into 10 clusters (Fig. 1a).

Recognising the role of homologous recombination as a dominant, recurring force in the continuing genomic evolution of E. faecium, the assignment of isolates to 


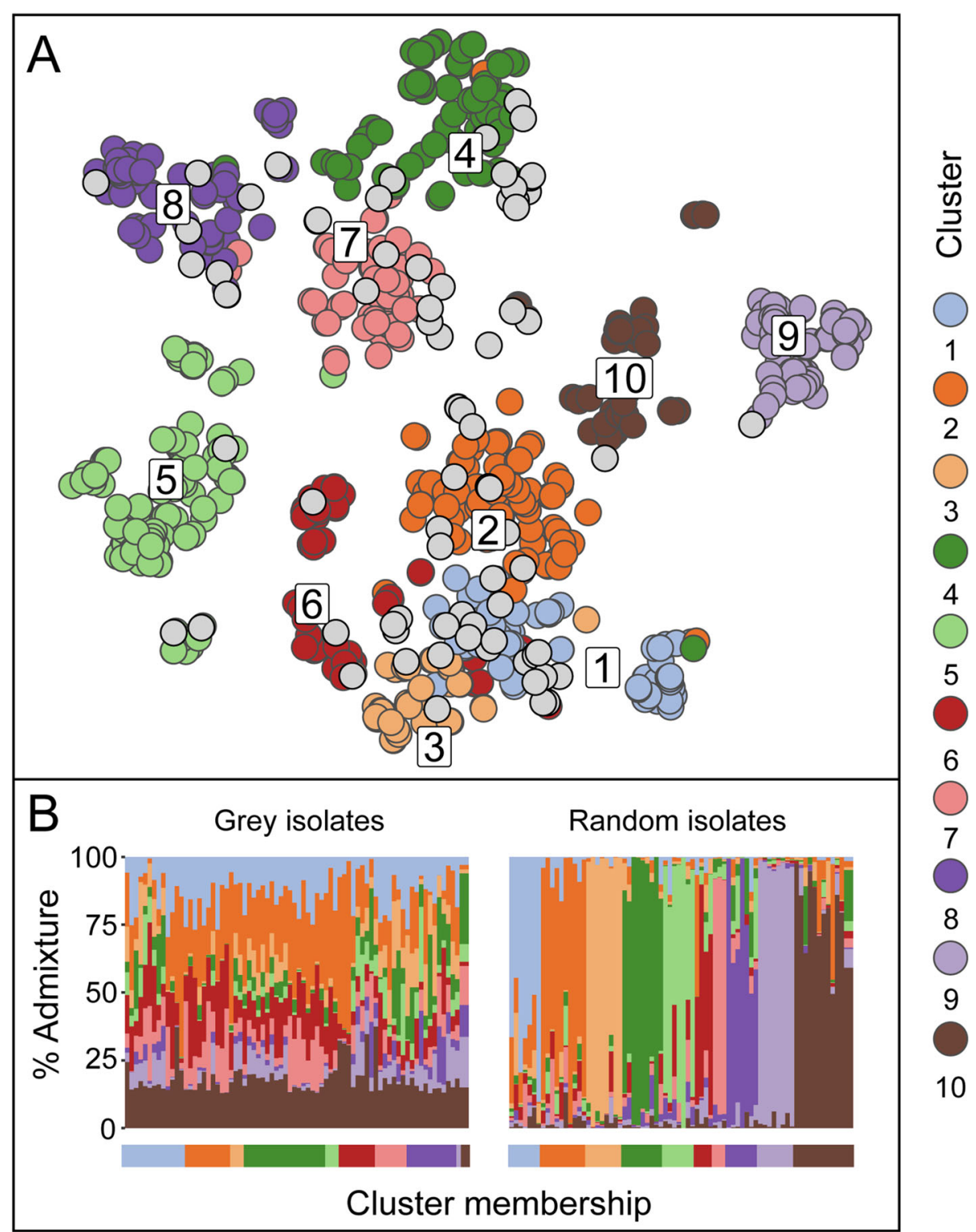

Fig. 1 Clustering among global A1 isolates. a Clustering of 973 E. faecium group A1 isolates based on the presence or absence of genes within the pan-genome identified using Panaroo. Labelled clusters are represented on a reduced dimensionality 2-D grid with member isolates coloured as shown in the legend. At a core-genome SNP level isolates within the same cluster are expected to share the same ancestry across the majority of their genomes. Using ChromoPainter, substantial core-genome admixture was detected in the 78 grey-shaded isolates, resulting in their exclusion from the designated pan-genome clusters. $\mathbf{b}$ The levels of admixture of the aforementioned 78 grey-shaded isolates in $\mathbf{a}$ (left) are contrasted with estimated admixture of 78 randomly chosen isolates from the remaining 895 isolates. The $x$-axis label shows the initial cluster assignment based on the pan-genome with the $y$-axis bars representing co-ancestry signals originating from other clusters, using the same colours as in a

pan-genome clusters was refined using chromosome painting [25]. This analysis traces the signal of coancestry shared between isolates, based on reads mapped to a reference core genome sequence of 1926 genes (those present in $>95 \%$ of isolates), and provides a "fingerprint" that distinguishes all but the most closely related isolates. Chromosome painting identified 78 isolates, typically visualised at the edges of the pangenome clusters (Fig. 1a, b), whose core genomes revealed substantial ancestry in multiple clusters. For the remaining 895 isolates, aggregating fragments of sequence including those transferred between lineages, a mean of $81 \%$ (IQR $75-89 \%$ ) of an isolate's core genome shared co-ancestry with other isolates within the cluster. 


\section{Pan-genome and plasmidome}

Panaroo identified 15,788 genes and gene variants among A1 isolates, with a median genome size of 2781 identifiable genes. Along with 1926 genes present in > 95\% of isolates, 2084 "shell" genes were found in 10$95 \%$ of genomes, arranged in groups of similar frequency hinting at assemblages of genes shared across multiple clusters. These estimates were recapitulated using Roary [22] and are larger than previously reported, indicating the influence of non-human isolates in limiting coregenome size estimates in other studies [10]. A substantial proportion $(854 ; 40.9 \%)$ of genes were identified on plasmids (Additional file 2: Figure S1), providing evidence of plasmid gain contributing to cluster emergence and diversification with 129 of the 366 cluster-associated genes occurring on a plasmid in at least one isolate (Fig. 2). The total frequencies of plasmid-derived genes were similar across clusters. Most of the remaining 11, 778 genes $(76 \%$ of the total) were found in no more than a handful of closely related isolates $(<5 \%$ of isolates), reflecting in each case a probable single acquisition from an outside source. Although the average nucleotide diversity for core genes was low ( $\mathrm{pi}=0.0056$ ); substantial variability, through the identification of homologues with $>5 \%$ divergence, in some ubiquitous genes including housekeeping genes ( $a d k, a t p A$ and $p s t S)$ was noted. These patterns likely indicate a single clonal expansion leading to A1 with infrequent replacement of functionally constrained genes from outside the E. faecium species.

\section{Patterns of $\mathrm{A} 1$ dissemination and admixture}

Genome-wide nucleotide diversity among A1 E. faecium isolates is small, stemming from its origin as a hospitalrestricted clone. Nevertheless, it is possible to detect core genome lineages that have arisen by a combination of mutation and admixture with non-A1 E. faecium. Of the 10 clusters, some $(9,10)$ were found almost entirely in one geographic location (country), while others were limited to geographical regions (clusters 3 and 4 in Asia and clusters 5 and 7 in Europe). Samples in the remaining clusters $(1,2,6,8)$ originated from highly overlapping, indistinct territories within Europe or internationally (Additional file 2: Figure S2). The genome sequences assigned to each of the generalised European/ world clusters typically include many of the oldest samples (especially cluster 2) in contrast with geographically specific clusters (Additional file 2: Figure S3). The relationship between time-depth of sampling and diversity suggests that A1 has evolved and disseminated over timescales comparable with sampling times, i.e. decades.

We next focus on three selected clusters to examine and illustrate distinct patterns of adaptation, admixture between clusters and networks of spread. Cluster 5

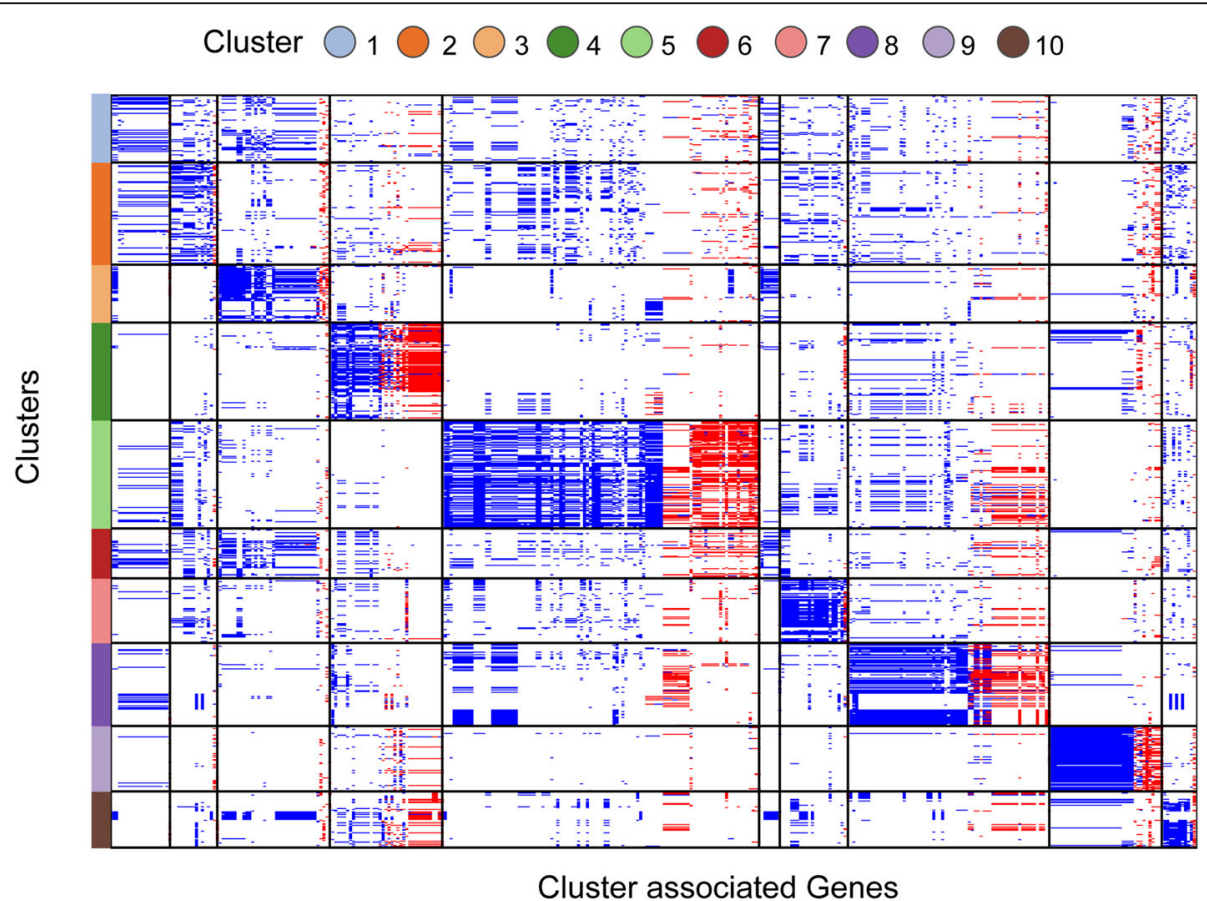

Fig. 2 Cluster-associated genes. All 366 genes significantly associated $(p<0.05)$ with pan-genome clusters are depicted with chromosomal and plasmid-derived genes coloured blue and red respectively. Of the 366 cluster-associated genes, 129 genes occurred on a plasmid in at least one isolate. Genes (along the $x$-axis) are grouped by cluster (along the $y$-axis) as depicted in the legend. No gene was exclusively limited to any one cluster, with the largest complement of genes associated with cluster $5(n=107)$ 
emerged in Denmark before disseminating across Northern Europe (Fig. 3). The single Australian isolate within cluster 5 is suggestive of wider spread; however, the identified expansion seems predominantly restricted to mainland Europe. Substantial recombination (involving all core genes) has resulted in highly variable patterns of admixture at the gene level. However, isolates from a single country retain a strong recent clonal signature inclusive of the pan-genome (depicted in panels A and B in Fig. 3), superimposed on a deeper structure of emergence, adaptation and expansion prior to spread. The scatter of Dutch isolates, for example, across the cluster phylogeny, reveals a pattern of homogenisation across Europe, leading to a picture of the co-circulation of multiple subclones that could not previously be appreciated; these co-circulating lineages augment the pool of coregenome variants and accessory genes that can contribute to the emergence of new lineages (Fig. 3).

To appreciate the granularity of the patterns of admixture and genetic exchange between clusters and isolates, focus is switched to cluster 9, consisting exclusively of isolates from Australia. The collection of cluster 9 isolates in multiple institutions in several cities rules out a single outbreak or clonal expansion event as a simple explanation for this cluster (data not shown). Signals of recombination are observed across all genes, incorporating sequences characteristic of all other clusters, in spite of the low frequencies of some clusters in Australia. In fact, local isolates provided the best match for a large proportion (53\%) of copying events from other clusters (Fig. 4), confirming the duality of local acquisition of sequences and links to the worldwide store of core genome variation. Sampling dates for cluster 9 were closely spaced, obscuring the relationship between time and diversification while remaining consistent with a rapid emergence.

Cluster 2, present in all sampled continents, was chosen to examine the characteristics and routes of global dissemination (Fig. 5). A phylogenetic analysis after masking recombination revealed a similar substructure as in cluster 5 , in which clonal patterns are limited by location and blurred by admixture patterns (Additional file 2: Figure S3). Given the considerable spread and time depth of European isolates, the phylogeny is most consistent with introduction(s) of E. faecium into the USA from Europe, most probably from the UK. The lack of a consistent pattern of vancomycin resistance genes among most closely linked isolates in different countries suggests the acquisition of vancomycin resistance at a local level (Fig. 6) [9]. Cluster 2's epicentre remains in Europe, with regional links between Japan and Australia, and North and South America.

\section{Antimicrobial resistance and virulome}

The availability of a globally representative collection of E. faecium A1 isolates facilitated a survey of genes associated with antimicrobial resistance (AMR). A

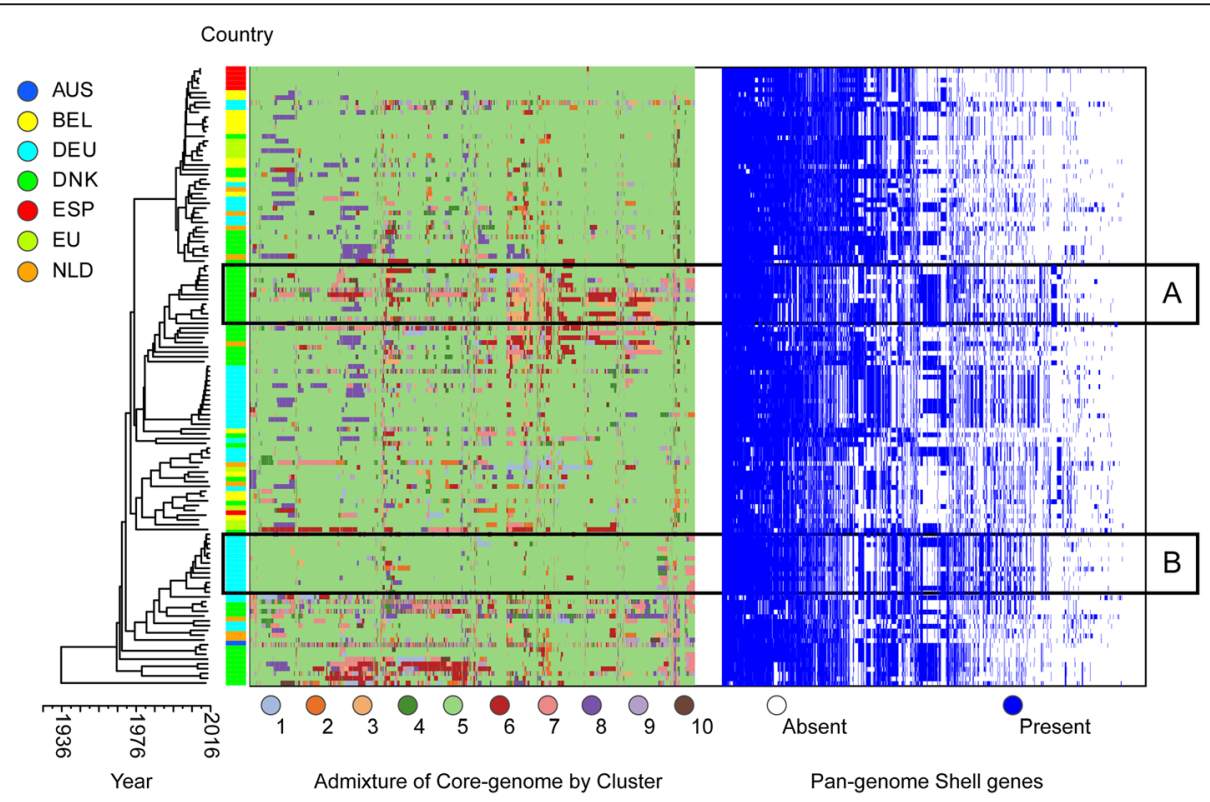

Fig. 3 Cluster 5: Evidence of local adaptation and regional dissemination. Maximum clade credibility tree of 129 cluster 5 isolates following masking of recombination. The first column to the right of the tree is coloured by sampled country (key left upper corner: AUS, Australia; BEL, Belgium; DEU, Germany; DNK, Denmark; ESP, Spain; EU, European Union; NLD, Netherlands). The subsequent column or heatmap depicts admixture events across the core genes of isolates coloured by "donating" cluster (key below heatmap). Similar background patterns of admixture are observed, accompanied by evidence of ongoing recombination at regional levels. Two such instances linked to Denmark and Germany are highlighted by boxes A and B respectively. Time scale is shown on the $y$-axis below the phylogeny 


\section{Donor Proportion}

By Country

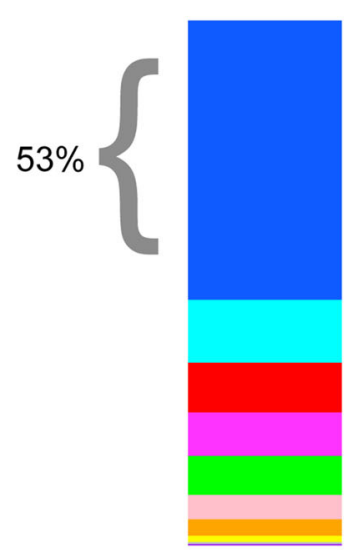

By Cluster

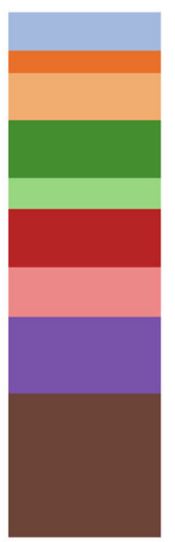

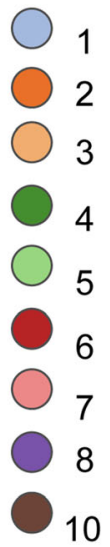

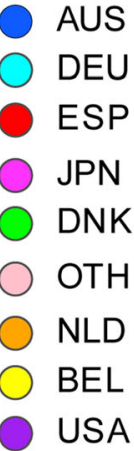

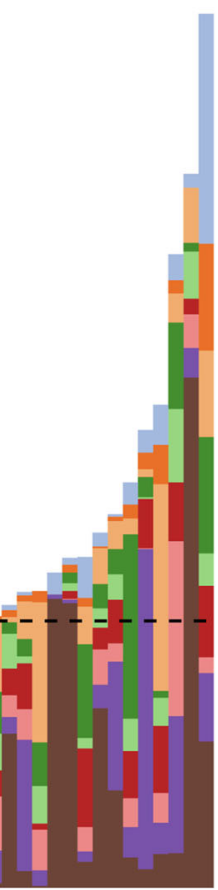

\section{Cluster 9 Isolates}

Fig. 4 Cluster 9. The bottom panel along the $x$-axis represents individual cluster 9 isolates (originating exclusively from Australia) with coloured vertical bars showing individual admixture signals across their core-genome by donor cluster. The dashed line depicts an admixture threshold of 10\%. The top half of the panel reflects the proportions of admixed regions by donor country (left) and donor cluster (right). Overall, cluster 9 isolates share the majority of their genome co-ancestry (between $61 \%$ and 98\%) with other cluster 9 isolates. In admixed regions, the best genomic match to the donor cluster originated from another Australian isolate in $53 \%$ of cases

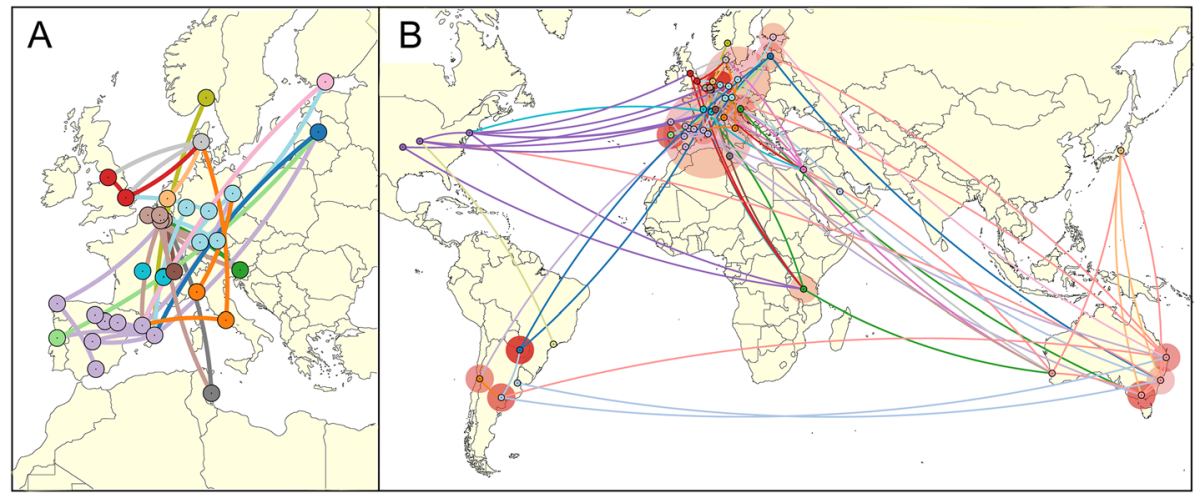

OARG OAUS OBEL OBRA OCHI ODEU ODNK OESP OFIN OFRA OGBR OISR OITA OJAP OLVA ONLD ONOR OPRG OPRT OSAR OSVN OSWI OTUN OTZA OUSA

Country Legend

Fig. 5 Dissemination routes of E. faecium across the world. Worldwide dissemination of genomes belonging to cluster $2(n=122)$ analysed using spatial, temporal and genomic data through BEAST v2.6 and visualised using SpreaD3. Coloured nodes on the map represent an isolate's country of origin (as shown in the legend), while connecting lines are coloured by destination location. An expanded view is shown in panel a to show inferred spread of E. faecium isolates across Europe with complex links between and within countries. b E. faecium dissemination across the globe with surrounding circle sizes proportional to the number of lineages (isolates that share the same continuous line of ancestry) that occur in that location and captures the absolute and relative intensity of the local spread at any given point in time 


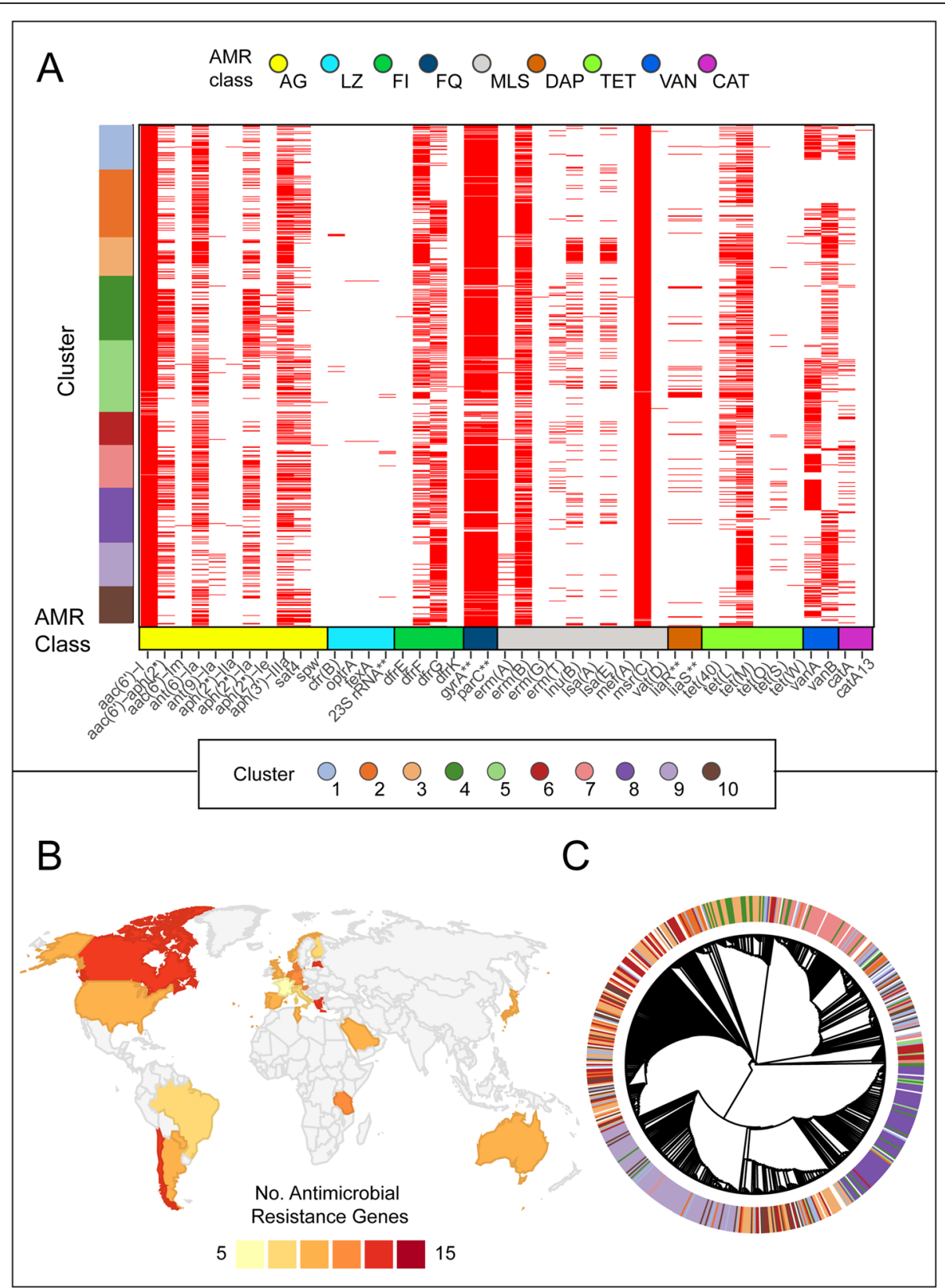

Fig. 6 Antimicrobial Resistance. a Presence (red) or absence (white) of resistance genes grouped by isolates within Panaroo clusters along the $y$ axis. Genes are named along the $x$-axis with mutational resistance represented by gene names highlighted by 2 asterisks and grouped by AMR class. AG, aminoglycosides; LZ, linezolid; FI, folate inhibitors; FQ, fluoroquinolones; MLS, macrolide-lincosamide-streptogramin; DAP, daptomycin; TET, tetracycline; VAN, vancomycin; and CAT, chloramphenicol. b Heatmap of the average number of antimicrobial resistance genes resulting in resistance within isolates by country. No isolates were included from grey shades countries. c Circular dendrogram of the tetM gene with designated cluster by colour depicted in the outer ring. Evidence of clustering is still seen (e.g. clusters 7, 8 and 9) but extensive transfer of alleles between clusters by horizontal gene transfer is evident

vancomycin resistance gene cluster was present in 544 (56\%; 261 vanA, 283 vanB) A1 isolates. This study recapitulated known associations between country and mode of vancomycin resistance. Cluster 9 (predominantly Australian) and cluster 7 (predominantly German) were skewed towards $v a n B$, while cluster 6 (European countries) was significantly more likely to harbour $\operatorname{van} A$ ( $p<$
0.05) (Fig. 6a). The cluster 9 correlation is driven by the dominance of a single multi-locus sequence type, ST796 in that cluster (Additional file 1: Table S1), whereas in clusters 6 and 7 multiple acquisitions of vancomycin resistance are implicated. Thus, the association of van type with geography may be in part driven by the short-term success of particular lineages of $E$. faecium. 
Trimethoprim resistance, encoded by the $d f r G$ gene, was similarly clonally associated (data not shown). Less clonal and more dynamic patterns were observed for tetM (tetracycline resistance) and aminoglycoside resistance genes, with evidence for loss events and acquisition of multiple distinct gene variants within a cluster.

Several non-synonymous variants in the liaSFR gene cluster, including the daptomycin resistance mutations, LiaR $^{\mathrm{T} 73 C}$ and LiaS ${ }^{\mathrm{T} 120 \mathrm{~A}}$ (CARD database) [35], were observed in our collection. Resistance mutations were carried together and emerged from only one of the two common allele backgrounds (Additional file 2: Figure S4), which were positively associated with clusters 1,3 and $6(p<0.05)$. This background was detected in isolates from most sampled countries studied, implying the emergence of daptomycin resistance in most locations, subject to local selection pressure. Although daptomycin is generally used when treating a VRE infection, daptomycin resistance was not associated with vancomycin resistance in our sample set.

The G2576T mutation in 23S rRNA associated with linezolid resistance was detected in 7 isolates, but, unlike daptomycin, no association with clusters could be detected. Plasmid-mediated linezolid resistance was found in two isolates harbouring the optrA gene in conjunction with $f e x A$ (florfenicol-chloramphenicol transporter gene) and in 6 isolates containing the $\operatorname{cfr}(\mathrm{B})$ gene.

A1 E. faecium isolates carry a median 10 (range 5-15) resistance genes for which country- and region-specific (notably European) frequencies are observed (Fig. 6b). Overall, E. faecium tends to be multi-resistant, harbouring quinolone mutations (in both $\operatorname{parC}$ and gyrA genes) and at least one gene conferring resistance to aminoglycoside, macrolide-lincosamide-streptogramin, tetracycline and antifolate antimicrobials. Over the study period, hospital-associated A1 E. faecium has become more resistant, from a median complement of 4 genes in 1986 to 11 in 2016 (Additional file 2: Figure S5). At the gene level, there is evidence of extensive horizontal gene transfer, in the form of multiple clonal backgrounds within and shared between pan-genome clusters. One such example, the tetM gene, depicted in Fig. 6c, illustrates a continuum from minimal admixture and ongoing cluster specificity (clusters 7, 8 and 9) to frequent exchanges resulting in loss of cluster signals.

Ten putative virulence genes were detected in the A1 E. faecium isolates. Of these, 6 were found in $>75 \%$ of isolates and included genes associated with biofilm formation (bopD), adhesion $(\operatorname{sgr} A)$, bile salt hydrolase (bsh), collagen binding $(a c m, c l p P)$ and metal transporter (psaA) genes (Additional file 2: Figure S6). The capsular polysaccharide gene (cpsF) was present in less than $1 \%$ of isolates and is generally not associated with virulence in E. faecium. Differences in virulomes by cluster were typically characterised by the reduced proportion of isolates carrying specific virulence genes. The functional consequences of these differences are unknown. Similarly, whether these observations represent gene loss or gain events is unknown as no specific patterns were observed in relation to time, geography or country of origin.

\section{Discussion}

The A1 clade of E. faecium has a worldwide distribution in which it dominates the hospital population of diseasecausing E. faecium in each location where it has been studied. The low levels of nucleotide diversity among genes common to all isolates are consistent with the emergence of A1 from the expansion of a single clone, with substantial continuing evolution, during the past several decades. The two main modes of genomic evolution that continue to shape E. faecium A1's diversity and population structure are the acquisition and loss of assemblages of genes that form the mobilizable genome (through the acquisition of mobile genetic elements including plasmids), and homologous recombination of the core genome, in which clones that have emerged since A1's formation are recombined, eroding initial signals of clonality and in some identifiable cases producing new clonal clusters. Both modes occur at large scales and with considerable tempo, complicating large-scale, genome-wide analyses of population history and structure.

In this study of the largest available collection of diverse and globally distributed E. faecium genome sequences from hospitalised patients, comprising 973 A1 isolates from 31 countries collected over 30 years, we define a substantial pan-genome. The core genome contained nearly 2000 genes, accompanied by 854 common accessory genes encoded on plasmids (the "shell genome"), plus a much larger number of sporadically captured genes found in no more than a few isolates. Several gene patterns appear to have been acquired independently more than once, leading to geographically specific clusters and providing a strong indication of ongoing interactions among A1 Enterococci in the gastrointestinal tracts of patients, where many of the same genes are recycled into different clusters. Sporadic acquisitions indicate that hospital E. faecium also retains access to a much larger pool of potential genes that have failed to become established in successful clusters.

Within each pan-genome cluster, we were able to identify one or more dominant lineages defined as common haplotypes on which are superimposed imported sequences, acquired through homologous recombination, whose origins are in other clusters. These isolates include ones whose core genomes place them mid-way between established lineages (e.g. the grey-coloured 
isolates depicted in Fig. 1) and provide the potential starting point for new clusters. These patterns reveal a continuing process of formation of clones, over relatively short periods (decades), comprising newly recombined genomes, some of which are then locally or regionally successful while continuing to recombine and gain and lose genes.

A recent analysis of a globally representative sample set estimated the emergence of the hospital-associated A1 E. faecium at $~ 302$ years ago [36], in marked contrast with estimates as recent as 74 years [8]. This discrepancy is difficult to resolve using existing methods, since accurate phylogenomic analyses rely on the assumptions that recombination occurs across a restricted part of the genome and that these regions can be reliably detected and removed. Our analysis reveals recombination across the whole genome, even in relatively small sample subsets. Consequently, molecular clock approaches are prone to inaccuracies, providing an explanation for wide discrepancies in estimates. Leaving aside phylogenetic analyses, the very low diversity of large parts of the genome and the global distribution of A1 genomes is consistent with the emergence of E. faecium A1 over recent decades, followed by global dissemination.

In contrast with a lack of precise information about its date of emergence, our analysis yields some observations relevant to $E$. faecium A1's origins. The A1 population in Europe contains more distinct lineages (clusters) than in other sampled locations, and this is reflected in the evidently complex history of recombination in cluster 2 . In contrast, other well-sampled locations such as Australia reveal a less complex, more geographically specific pattern of sequence exchange. These observations are consistent with Europe as the birthplace of and longest established home of A1, a conclusion that needs to be tested, given the limited number of American isolates in our study and the potential resulting sampling bias.

Our improved understanding of the processes through which A1 E. faecium continues to evolve may help us to better understand the dynamics of selectable traits such as antimicrobial resistance. Our analysis suggests that gain and loss of van elements (either the vanA or the $\operatorname{van} B$ cassette) leading to vancomycin resistance is common enough that geographic specificities are an accident of history, driven by chance through the acquisition and spread of a particular cassette in a particular population. Rather than reflecting a genetic propensity to carry a particular van type, common van loss events are likely to be followed by re-acquisition of the same van type simply because it happens to be frequent in that particular population [2,37]. This pattern (that gene acquisition is most likely to reflect the distribution of circulating genes) is mirrored by the accessory genome as a whole and explains how a particular complement of shell genes (a pan-genome cluster) exists without being completely linked to a set of core genome lineages. The implication of these observations is that the raw materials for phenotypic plasticity may be population-specific in the short term, but subject to global dissemination and mixing over longer timescales.

Our study also reveals the distinct dynamics underlying resistance to the last-line antibiotic, daptomycin. The variants responsible for daptomycin resistance only appear on one of two ancestral forms of the liaS gene that are associated with clusters and geography but nevertheless appear in all populations, implying that mutational selection for resistance is only possible in some infections, but the requisite genotypic background may eventually spread by recombination combined with selection.

\section{Conclusions}

The patterns of variation that we describe for A1, the main hospital-adapted lineage of E. faecium, illustrate the ease with which new phenotypes are likely to continue to emerge, driven by local variations in selection and access to distinct gene pools via both homologous recombination and an extensively mobilizable pangenome. These genomic features suggest that controlling the hospital spread of $E$. faecium will remain challenging.

\section{Supplementary Information}

The online version contains supplementary material available at https://doi. org/10.1186/s13073-021-00868-0

Additional file 1: Table S1. Details of E. faecium short read sequence data used in the analysis.

Additional file 2: Figure S1. Plasmid and Chromosomal associated shell genes. Figure S2. Distribution of clusters by country and collection dates. Figure S3. Local adaptation and regional dissemination of $E$. faecium. Figure S4. LiaFSR haplotypes. Figure S5. Antibiotic resistance gene accumulation over time. Figure S6. Virulome.




We wish to acknowledge those responsible for all aspects of isolate curation, data collection, typing and sequencing at the different locations. Dr. A. P. Tedim from Spain, Dr. P. Worning from Denmark, Dr. J. K. Bender from Germany and Dr. A. Beukers from Australia.

\section{Authors' contributions}

Providing sequence data: SVH, RW, AH, SB, TC, KH, HW, BH, SK, GW, KY, AE, KR. Experimental design: SVH, RW, JC, RB. Analysis: SVH, JC, RB. Manuscript preparation: SVH, RB. All authors read and approved the final manuscript.

\section{Funding}

No specific funding was obtained for this study. Individual authors have received funding as outlined below. R.J.L.W. was supported by the Joint Programming Initiative in Antimicrobial Resistance (JPIAMR Third call, STAR CS, JPIAMR2016-AC16/00039). TMC was supported by the European Commission (JPIAMR - STARCS project); InGEMICS-C (S2017/BMD-3691), funded by Comunidad de Madrid (Spain); CIBERESP (CIBER in Epidemiology and Public Health; CB06/02/0053), and grants AC16/00039 and Pl18/01942, integrated in the Spanish 2013-2016 and 2017-2020 R+D+I State Plans and co-funded by Instituto de Salud Carlos III and the European Regional Development Fund (ERDF, "A way to achieve Europe"). SMK acknowledges funding as part of the Methusalem-Excellence consortium VAX-IDEA, and JPIAMR-STARCS (JPIAMR2016-AC16/00039). AE was supported in part by federal funding from the National Institute of Allergy and Infectious Diseases, National Institutes of Health, Department of Health and Human Services, under Contract No. HHSN272200900018C and Grant No. U19Al1 10818 to the Broad Institute. JC was supported by the ERC grant no. 742158. RB benefited from Victorian State Government Operational Infrastructure Support and Australian Government NHMRC IRIISS to the Walter and Eliza Hall Institute of Medical Research and core funding to the Wellcome Centre for Human Genetics provided by the Wellcome Trust (award 203141/Z/16/Z).

\section{Availability of data and materials}

All new short read sequence data $(n=321)$ was uploaded to NCBI under project number PRJNA63689, https://www.ncbi.nlm.nih.gov/sra/PRJNA636894 [13]. The remaining $(n=826)$ sequences were downloaded from NCBI with project numbers and associated metadata provided in Additional file 1: Table S1.

\section{Declarations}

Ethics approval and consent to participate

Not applicable

\section{Consent for publication}

Not applicable

\section{Competing interests}

The authors declare that they have no competing interests.

\section{Author details}

${ }^{1}$ Department of Infectious Disesase and Microbiology, Royal Prince Alfred Hospital, Sydney, NSW, Australia. ${ }^{2}$ University of Sydney, Sydney, NSW, Australia. ${ }^{3}$ Department of Medical Microbiology, University Medical Center Utrech, Utrecht, The Netherlands. ${ }^{4}$ Cambridge University Hospitals NHS Foundation Trust, Cambridge, UK. ${ }^{5}$ Microbiological Diagnostic Unit Public Health Laboratory, The University of Melbourne at The Peter Doherty Institute for Infection and Immunity, Melbourne, Victoria, Australia. ${ }^{6}$ Department of Microbiology, Ramón y Cajal University Hospital and Ramón y Cajal Health Research Institute (IRYCIS), Madrid, Spain. ${ }^{7}$ Network Research Centre for Epidemiology and Public Health (CIBERESP), Madrid, Spain. ${ }^{8}$ Statens Serum Institut, 2300 Copenhagen S, Denmark. ${ }^{9}$ Department of Microbiology and Infection Control, Norwegian National Advisory Unit on Detection of Antimicrobial Resistance, University Hospital of North-Norway, Tromsø, Norway. ${ }^{10}$ Research Group for Host-Microbe Interactions, UiT - the Arctic University of Norway, Tromsø, Norway. ${ }^{11}$ MRSA Knowledge Center, Department of Clinical Microbiology, Hvidovre Hospital, Hvidovre, Denmark. ${ }^{12}$ Laboratory of Medical Microbiology, Vaccine \& Infectious Disease Institute, Universiteit Antwerpen, Wilrijk, Belgium. ${ }^{13}$ National Reference Centre for Staphylococci and Enterococci, Division of Nosocomial Pathogens and Antibiotic Resistances, Department of Infectious Diseases, Robert Koch Institute, Wernigerode Branch, Wernigerode, Germany. ${ }^{14}$ Department of
Laboratory Medicine, Nagasaki University Graduate School of Biomedical Sciences, Nagasaki, Japan. ${ }^{15}$ Infectious Disease \& Microbiome Program, Broad Institute, Cambridge, MA, USA. ${ }^{16}$ Department of Medicine, University of Cambridge, Cambridge, UK. ${ }^{17}$ Department of Biostatistics, University of Oslo, Oslo, Norway. ${ }^{18}$ Parasites and Microbes, Wellcome Sanger Institute, Hinxton, Cambridge, UK. ${ }^{19}$ The Walter and Eliza Hall Institute of Medical Research, Parkville, Victoria 3052, Australia. ${ }^{20}$ Department of Medical Biology, University of Melbourne, $1 \mathrm{G}$ Royal Parade, Melbourne, Victoria, Australia. ${ }^{21}$ Wellcome Centre for Human Genetics, University of Oxford, Roosevelt Drive, Oxford OX3 7BN, UK.

Received: 23 September 2020 Accepted: 15 March 2021

Published online: 30 March 2021

\section{References}

1. Weiner LM, Webb AK, Limbago B, Dudeck MA, Patel J, Kallen AJ, Edwards $J R$, Sievert DM. Antimicrobial-resistant pathogens associated with healthcare-associated infections: summary of data reported to the National Healthcare Safety Network at the Centers for Disease Control and Prevention, 2011-2014. Infect Control Hosp Epidemiol. 2016;37(11):1288-301. https://doi.org/10.1017/ice.2016.174.

2. van Hal SJ, Ip CLC, Ansari MA, Wilson DJ, Espedido BA, Jensen SO, et al. Evolutionary dynamics of Enterococcus faecium reveals complex genomic relationships between isolates with independent emergence of vancomycin resistance. Microb Genom. 2016;2(1):e000048. https://doi.org/10.1099/mgen. 0.000048 .

3. van Hal SJ, Beukers AG, Timms VJ, Ellem JA, Taylor P, Maley MW, Newton PJ, Ferguson JK, Lee A, Chen SC, Sintchenko V. Relentless spread and adaptation of non-typeable vanA vancomycin-resistant Enterococcus faecium: a genome-wide investigation. J Antimicrob Chemother. 2018;73(6): 1487-91. https://doi.org/10.1093/jac/dky074.

4. Bender JK, Kalmbach A, Fleige C, Klare I, Fuchs S, Werner G. Population structure and acquisition of the vanB resistance determinant in German clinical isolates of Enterococcus faecium ST192. Sci Rep. 2016;6(1):21847. https://doi.org/10.1038/srep21847.

5. Ayobami O, Willrich N, Reuss A, Eckmanns T, Markwart R. The ongoing challenge of vancomycin-resistant Enterococcus faecium and Enterococcus faecalis in Europe: an epidemiological analysis of bloodstream infections. Emerg Microbes Infect. 2020;9(1):1180-93. https://doi.org/10.1080/22221 751.2020.1769500

6. Bender JK, Cattoir V, Hegstad K, Sadowy E, Coque TM, Westh H, Hammerum AM, Schaffer K, Burns K, Murchan S, Novais C, Freitas AR, Peixe L, del Grosso M, Pantosti A, Werner G. Update on prevalence and mechanisms of resistance to linezolid, tigecycline and daptomycin in enterococci in Europe: towards a common nomenclature. Drug Resist Updat. 2018;40:25-39. https://doi.org/10.1016/j.drup.2018.10.002.

7. Miller WR, Munita JM, Arias CA. Mechanisms of antibiotic resistance in enterococci. Expert Rev Anti-Infect Ther. 2014;12(10):1221-36. https://doi. org/10.1586/14787210.2014.956092.

8. Lebreton F, van Schaik W, Manson McGuire A, Godfrey P, Griggs A, Mazumdar $V$, et al. Emergence of epidemic multidrug-resistant Enterococcus faecium from animal and commensal strains. mBio. 2013;4(4):e00534-13.

9. Raven KE, Gouliouris T, Brodrick H, Coll F, Brown NM, Reynolds R, Reuter S, Török ME, Parkhill J, Peacock SJ. Complex routes of nosocomial vancomycin-resistant Enterococcus faecium transmission revealed by genome sequencing. Clin Infect Dis. 2017;64(7):886-93. https://doi.org/10.1 093/cid/ciw872.

10. Arredondo-Alonso S, Top J, McNally A, Puranen S, Pesonen M, Pensar J, et al. Plasmids shaped the recent emergence of the major nosocomial pathogen Enterococcus faecium. mBio. 2020;11(1):e03284-19. https://doi. org/10.1128/mBio.03284-19.

11. Didelot $X$, Maiden MC. Impact of recombination on bacterial evolution. Trends Microbiol. 2010;18(7):315-22. https://doi.org/10.1016/j.tim.2010.04. 002.

12. Posada D, Crandall KA. The effect of recombination on the accuracy of phylogeny estimation. J Mol Evol. 2002;54(3):396-402. https://doi.org/10.1 007/s00239-001-0034-9.

13. van Hal SJ, Willems RJL, Gouliouris T, Ballard SA, Coque TM, Hammerum AM, et al. The Global dissemination of hospital clones of Enterococcus faecium. BioProject PRJNA636894 NCBI Sequence Read Archive. 2021 https://www. ncbi.nlm.nih.gov/sra/PRJNA636894. 
14. Bolger AM, Lohse M, Usadel B. Trimmomatic: a flexible trimmer for Illumina sequence data. Bioinformatics. 2014;30(15):2114-20. https://doi.org/10.1093/ bioinformatics/btu170.

15. Li H, Durbin R. Fast and accurate short read alignment with burrowswheeler transform. Bioinformatics. 2009;25(14):1754-60. https://doi.org/10.1 093/bioinformatics/btp324.

16. Garrison EMG. Haplotype-based variant detection from short-read sequencing. arXiv preprint arXiv:12073907 [q-bioGN]. 2012.

17. Cheng L, Connor TR, Sirén J, Aanensen DM, Corander J. Hierarchical and spatially explicit clustering of DNA sequences with BAPS software. Mol Biol Evol. 2013;30(5):1224-8. https://doi.org/10.1093/molbev/mst028.

18. Davis MP, van Dongen S, Abreu-Goodger C, Bartonicek N, Enright AJ. Kraken: a set of tools for quality control and analysis of high-throughput sequence data. Methods. 2013;63(1):41-9. https://doi.org/10.1016/j.ymeth.2 013.06.027.

19. Bankevich A, Nurk S, Antipov D, Gurevich AA, Dvorkin M, Kulikov AS, Lesin VM, Nikolenko SI, Pham S, Prjibelski AD, Pyshkin AV, Sirotkin AV, Vyahhi N, Tesler G, Alekseyev MA, Pevzner PA. SPAdes: a new genome assembly algorithm and its applications to single-cell sequencing. J Comput Biol. 2012;19(5):455-77. https://doi.org/10.1089/cmb.2012.0021.

20. Seemann T. Prokka: rapid prokaryotic genome annotation. Bioinformatics. 2014;30(14):2068-9. https://doi.org/10.1093/bioinformatics/btu153.

21. Tonkin-Hill G, MacAlasdair N, Ruis C, Weimann A, Horesh G, Lees JA, Gladstone RA, Lo S, Beaudoin C, Floto RA, Frost SDW, Corander J, Bentley $\mathrm{SD}$, Parkhill J. Producing polished prokaryotic pangenomes with the Panaroo pipeline. Genome Biol. 2020;21(1):180. https://doi.org/10.1186/s13 059-020-02090-4.

22. Page AJ, Cummins CA, Hunt M, Wong VK, Reuter S, Holden MT, et al. Roary: rapid large-scale prokaryote pan genome analysis. Bioinformatics. 2015; 31(22):3691-3. https://doi.org/10.1093/bioinformatics/btv421.

23. Krijthe J, van der Maaten L. Rtsne: T-Distributed Stochastic Neighbor Embedding using a Barnes-Hut Implementation. GitHub: https://github. com/jkrijthe/Rtsne. 2018.

24. Kassambara A, Mundt F. factoextra: Extract and Visualize the Results of Multivariate Data Analyses. GitHub: https://github.com/kassambara/fa ctoextra. 2020

25. Lawson DJ, Hellenthal G, Myers S, Falush D. Inference of population structure using dense haplotype data. PLoS Genet. 2012;8(1):e1002453. https://doi.org/10.1371/journal.pgen.1002453.

26. Didelot $X$, Wilson DJ. ClonalFrameML: efficient inference of recombination in whole bacterial genomes. PLoS Comput Biol. 2015;11(2):e1004041. https://doi.org/10.1371/journal.pcbi.1004041.

27. Bouckaert R, Vaughan TG, Barido-Sottani J, Duchene S, Fourment M, Gavryushkina A, et al. BEAST 2.5: an advanced software platform for Bayesian evolutionary analysis. PLoS Comput Biol. 2019;15(4):e1006650.

28. Bielejec F, Baele G, Vrancken B, Suchard MA, Rambaut A, Lemey P. SpreaD3: interactive visualization of spatiotemporal history and trait evolutionary processes. Mol Biol Evol. 2016;33(8):2167-9. https://doi.org/10.1093/molbev/ msw082.

29. Feldgarden M, Brover V, Haft DH, Prasad AB, Slotta DJ, Tolstoy I, et al. Validating the AMRFinder tool and resistance gene database by using antimicrobial resistance genotype-phenotype correlations in a collection of isolates. Antimicrob Agents Chemother. 2019;63(11):e00483-19. https://doi. org/10.1128/AAC.00483-19.

30. Bandelt HJ, Forster P, Rohl A. Median-joining networks for inferring intraspecific phylogenies. Mol Biol Evol. 1999;16(1):37-48. https://doi.org/1 0.1093/oxfordjournals.molbev.a026036.

31. Beukers AG, Hasman H, Hegstad K, van Hal SJ. Recommendations to address the difficulties encountered when determining linezolid resistance from whole-genome sequencing data. Antimicrob Agents Chemother. 2018; 62(8):e00613-18. https://doi.org/10.1128/AAC.00613-18.

32. Chen L, Yang J, Yu J, Yao Z, Sun L, Shen Y, Jin Q. VFDB: a reference database for bacterial virulence factors. Nucleic Acids Res. 2005;33(Database issue):D325-8. https://doi.org/10.1093/nar/gki008.

33. Arredondo-Alonso S, Rogers MRC, Braat JC, Verschuuren TD, Top J, Corander J, et al. mlplasmids: a user-friendly tool to predict plasmid- and chromosome-derived sequences for single species. Microb Genom. 2018; 4(11):e000224. https://doi.org/10.1099/mgen.0.000224.

34. Core Team R. R: a language and environment for statistical computing. Vienna: R Foundation for statistical computing; 2019. https://www.R-project. org/
35. The Comprehensive Antibiotic Resistance Database (CARD). Last accessed at https://card.mcmaster.ca in May 2020.

36. Rios R, Reyes J, Carvajal LP, Rincon S, Panesso D, Echeverri AM, Dinh A, Kolokotronis SO, Narechania A, Tran TT, Munita JM, Murray BE, Planet PJ , Arias CA, Diaz L. Genomic epidemiology of vancomycin-resistant Enterococcus faecium (VREfm) in Latin America: revisiting the global VRE population structure. Sci Rep. 2020;10(1):5636. https://doi.org/10.1038/s41 598-020-62371-7.

37. Howden BP, Holt KE, Lam MM, Seemann T, Ballard S, Coombs GW, et al. Genomic insights to control the emergence of vancomycin-resistant enterococci. mBio. 2013;4(4):00412-13. https://mbio.asm.org/content/4/4/ e00412-13.

\section{Publisher's Note}

Springer Nature remains neutral with regard to jurisdictional claims in published maps and institutional affiliations.
Ready to submit your research? Choose BMC and benefit from:

- fast, convenient online submission

- thorough peer review by experienced researchers in your field

- rapid publication on acceptance

- support for research data, including large and complex data types

- gold Open Access which fosters wider collaboration and increased citations

- maximum visibility for your research: over $100 \mathrm{M}$ website views per year

At BMC, research is always in progress.

Learn more biomedcentral.com/submissions 